\title{
Tree-based protection of multicast services in WDM mesh networks
}

\author{
Long Long Ahmed E. Kamal \\ Dept. of Electrical and Computer Eng., Iowa State University, Ames, IA 50011, U.S.A. \\ E-mail: \{longlong, kamal\}@iastate.edu
}

\begin{abstract}
In this paper we address the multicast survivability problem of using minimum resources to provision a multicast session and its protection paths (trees) in a network such that the session is protected against any single-link failure. We propose a new protection scheme, namely, Segment-based Protection Tree (SPT). In SPT scheme, a given multicast session is first provisioned as a primary multicast tree, and then each segment on the primary tree is protected by a multicast tree instead of a path, as in most existing approaches. We also analyze the recovery performance of SPT and design a Reconfiguration Calculation Algorithm to compute the average number of reconfigurations upon any link failure. We study the performance of the SPT scheme in different traffic scenarios. The numerical results show that SPT outperforms the best existing approaches. It uses less than $10 \%$ extra resources to provision a survivable multicast session over the optimal solution and $0.2-4 \%$ lower than the existing approaches in the various traffic cases and has the average number of reconfigurations $10-86 \%$ less than the best cost efficient approach.
\end{abstract}

\section{INTRODUCTION}

Wavelength-division multiplexing (WDM) technology allows an aggregate traffic on the order of Tbps to be carried on a single fiber, with each wavelength carrying traffic in the tens of Gbps order. Such advances meet the explosive increase of bandwidth demand in the Internet and enable a great variety of network applications to be provisioned [1]. Several of these applications employ the multicast service mode, such as video distribution, online gaming and so on. To implement multicasting, a node should have the capability to replicate an incoming packet into multiple copies. In the context of optical networks, there are two ways to implement the multicast function at a node, unicast and multicast. In unicast mode, traffic duplication can only be implemented in the electronic domain, whereas in multicast mode, traffic duplication can be done in the optical domain by using optical splitters [5]. If a multicast session is provisioned as a tree in the optical domain, it is called a "light-tree" which originates at a source node and delivers the same data to a number of destination (leaf) nodes [3].

As the capacity of a fiber increases significantly, a fiber cut caused by an accident or a failure of a switch port or a node interface may lead to loss of tremendous amounts of data. In the scenario of multicast service, data loss on one fiber may cause the disruption of delivery to multiple nodes. Therefore, efforts have been exerted to deal with protection of a multicast

This research was supported in part by grant CNS-0626741 from the National Science Foundation. session against single link failure. A straightforward method proposed in [4] is to find two link disjoint light trees and both of them start from the source and end at the destination nodes. It is clear that this method is not capacity efficient since it is not always possible to find two link disjoint trees in a network. In [2], the authors introduced a number of protection schemes: link-based, segment-based and path-based. In linkbased and segment-based approaches, a multicast session is routed first to construct a multicast tree, and then each link or segment on the tree is protected by a path starting at the tail node and finishing at the head node of the link or segment it protects. Alternatively, a path-based protection scheme, named optimal path-pair-based shared disjoint paths (OPP_SDP) algorithm, achieves the best result in terms of network resource consumption in [2] by self-sharing primary and spare capacity [7]. The idea is to find two shortest link disjoint paths for each source and destination pair [13]. Recently, a couple of new technologies were applied to the survivability problem, $p$-cycle[8] and network coding[9]. They do have some nice features such as fast recovery speed of $p$-cycle or high bandwidth utilization of network coding. However, $p$ cycle-based schemes have to find cycles that traverse protected edges which may make the scheme less capacity efficient, while network coding introduces extra computational cost as well as O-E-O conversion since network coding can only be performed in the electronic domain in current optical networks, which may introduce an additional expense.

A path-based scheme, called multicast protection through spanning paths (MPSP), proposed in [6], outperforms OPP_SDP under both static and dynamic traffic patterns. It first provisions a primary multicast tree and then establishes a number of paths to protect each path between any pair of leaf nodes on the primary tree, called spanning path. Each path is link disjoint from the spanning path it protects. However, this scheme relies on the assumption that wavelengths reserved in a fiber can be used in two opposite directions by reconfiguring the switches at two end nodes. However, this feature cannot be achieved in practice. Between two connected nodes, there are usually two physical fibers set up and each of them works in one direction. The switches at end nodes use input and output ports to connect incoming and outgoing fibers, respectively [2], [5]. Reserved capacity (wavelength) in a fiber cannot be used in both directions by simply reconfiguring the switches at end nodes due to the fixed switching ports. One way to enable this feature is to change the physical infrastructure by deploying 
a pair of circulators between two nodes as shown in Fig. 1. The fiber is connected to the circulators instead of switching ports on the switches. The circulators connect to both input and output ports on the nearby switches and can configure the fiber to connect to either input port or output port. Only changing the configuration of both switches and circulators will make the transmission in both directions on the same fiber possible such that one unit of capacity reserved in a directed link can be shared by primary and protection path in MPSP scheme. Due to the infrastructure of current backbone networks, the lack of support for this functionality and the restrictions this imposes on other modes of communication, we do not take this assumption into consideration in our proposed scheme.

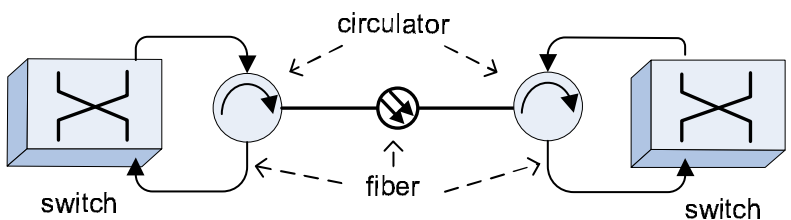

Fig. 1. Additional depolyment of circulators enables capacity sharing in opposite directions of a fiber

A tree-based protection scheme, segment-based protection tree (SPT) algorithm, is proposed in this paper to provision a multicast request and protect it against any single link failure. We first provision the multicast session on a light tree and then construct protection multicast trees instead of paths to protect the primary light tree. Each protection tree, similar to primary tree, is rooted at the source and reaches every destination in the session. Each segment on the primary tree is protected by a protection tree. A protection tree can share any link with the primary tree as well as other protection trees. The uniqueness of our schemes is that each protection tree is a complete multicast tree from source to destinations. It does not have to traverse the end nodes of a segment it protects. In this case, multiple segments may share one protection tree, which potentially improves the efficiency of the bandwidth utilization.

The rest of this paper is organized as follows. In Section II, we present the assumptions and statement of the problem addressed. The proposed scheme, SPT, will be introduced in Section III. The method of computing the average number of reconfigurations will be presented in Section IV. Numerical results will be presented and explained in Section V. Finally, we conclude this paper in Section VI.

\section{Problem Statement}

A typical multicast session is unidirectional whereas the links of a typical WDM mesh network are bidirectional, since each link has two optical fibers transporting signals in two opposite directions with the same capacity. Each directed fiber is also called "an arc" in [2]. Meanwhile, each arc is assigned a value to indicate the cost of transmitting the data from one end to the other. The cost usually refers to the length of the physical fiber.
Accordingly, we make the following assumptions and present the formal statements of the multicast protection problem:

1) Given a weighted directed connected graph $G=(V, E)$ in which each directed $\operatorname{link}^{1} e=(u, v) \in E$ where $u, v \in V$ is assigned a weight (cost) $c_{e}$ and a capacity with $W$ wavelengths. The graph, $G$, is at least 2connected.

2) Given a directed multicast request $D$ with a source node $s$ and a set of destinations $\left\{d_{1}, d_{2}, \ldots, d_{M}\right\}$ where $s, d_{i} \in$ $V$ and $M$ is the number of destination nodes. The traffic rate of the session is equal to one wavelength per unit time. $D$ is expressed as $\left(s,\left\{d_{1}, d_{2}, \ldots, d_{M}\right\}\right)$.

3) A single link failure will cut off the links in both directions such that traffic delivered in both fibers will be lost. Thus, when we claim two link-disjoint paths (trees) in this article, it indicates that two paths (trees) do not travel the links with the same end nodes in any direction.

4) In this article, we assume that each network node is equipped with an optical switch, optical splitters and wavelength converters if necessary.

The multicast protection problem is described as follow:

Given a weighted graph $G=(V, E)$ and a multicast request $D$, how can we utilize the minimum total cost to provision a given multicast session such that the multicast service is survivable against any single link failure in $G$ ?

\section{TREe-BASEd PRotection Schemes}

In this section, we present the tree-based protection scheme, SPT, to provision multicast requests against any single-link failure.

SPT scheme consists of two phases. The first phase is multicast tree construction, which finds a primary light-tree that delivers data from the source to all the destinations with the minimum cost. Deployment of optical splitters at each network node enables multicast implementation in the optical domain. Thus, this problem turns out to be a classic graph theory problem, "Steiner tree problem", which has been proven NP-complete [10]. Many approximate algorithms have been proposed in the literature such as Nearest Participant First (NPF) algorithm [11], KMB algorithm [12], pruned Prim's heuristic [13], referred to as PPH and so on. We actually consider three multicast schemes in the construction of multicast tree: NPF, pruned Prim's heuristic and simply using Dijkstra's Shortest Path algorithm, namely, DST, to find the shortest path from the source to each destination and combining all the paths to construct a multicast tree.

The heuristic NPF is a greedy-based algorithm with time complexity $O\left(M|V|^{2}\right)$. The procedure is explained as follow:

1) start from the source node;

2) find a destination node that is closest to the current tree;

${ }^{1}$ Here we use "link" to represent "arc" in [2] and therefore link $(u, v)$ and $(v, u)$ are two different links but have the same cost and capacity. 
3) connect the closest destination node to the closest part of the tree;

4) repeat until all the destinations are connected in the tree.

Prim's algorithm is a well known approach of finding the minimum spanning tree with time complexity $O\left(|V|^{2}\right)$. Based on the minimum spanning tree obtained, PPH trims the unwanted branches such that the resulting multicast tree only reaches the given destinations. The total time complexity is $O\left(|V|^{2}+M|V|\right)=O\left(|V|^{2}\right)$.

The algorithm DST, with time complexity $O\left(M|V|^{2}\right)$, is straightforward and is actually a special case of NPF by assuming that the source is the only node on the current tree. Thus, a multicast tree produced by DST always has equivalent or larger cost than what NPF produces. The reason to consider DST in phase one is that the objective function is the total cost of the final survivable multicast session other than that of the primary tree alone. A large primary multicast tree may require a small cost of protection trees to protect it, which can still end up with a good total cost.

In the second phase, we try to provision a topology to protect each primary tree obtained in phase one. Each tree is decomposed into a number of segments. Following the definition in [2], a segment is defined as the sequence of links from the source or any branch node (on a tree) to a leaf node or to a downstream branch node. For each segment of the tree, the SPT scheme establishes another multicast tree to protect it, called "protection tree". A protection tree is generated by running both NPF and PPH and selecting the one with the less network cost. We do not consider DST here because DST is a special case of NPF and can never produce better solution than NPF. Each protection tree must not traverse the segment it protects. However, it is not necessary for it to pass two end nodes of any segment it protects either. Any protection tree is a complete multicast tree rooted at the source and destined to all the destinations regardless of which segment it protects.

Several symbols used in the algorithms are explained in Table I:

TABLE I

LIST OF SYMBOLS

\begin{tabular}{|c|c|}
\hline Symbol & Meaning \\
\hline$T_{m_{k}}$ & $\begin{array}{l}\text { the } k \text { th primary multicast tree obtained by heuristic } k \text {, } \\
\text { where } 0 \leq k<3 \text { and } 0,1 \text { and } 2 \text { represents heuristic } \\
\text { algorithm NPF, PPH and DST, respectively }\end{array}$ \\
\hline$T_{p_{i}}^{k}$ & the $i$ th protection tree for primary multicast tree $k$ \\
\hline $\mathbf{P}_{k}$ & the union of all the protection trees, denoted by $\bigcup_{i} T_{p}^{k}$ \\
\hline $\mathbf{R}_{k}$ & $\begin{array}{l}\text { the union of all links used for the multicast session } \\
\text { generated by heuristic } k \text {, denoted by } T_{m_{k}} \cup \mathbf{P}_{k}\end{array}$ \\
\hline$c_{e}$ & the cost of link $e \in E$ \\
\hline
\end{tabular}

The SPT heuristic is presented in Algorithm 1.

The basic protection unit is "segment" on the primary tree, denoted by $l \in T_{m_{k}}$. If any existing protection tree established earlier does not traverse $l$ and its counterpart in the opposite direction $^{2}$, then $l$ is protected by this tree upon any failure of

\footnotetext{
${ }^{2}$ In the rest of the paper, when we say a tree does not travel a link or segment, it indicates that the tree does not travel the link or the segment in either direction
}

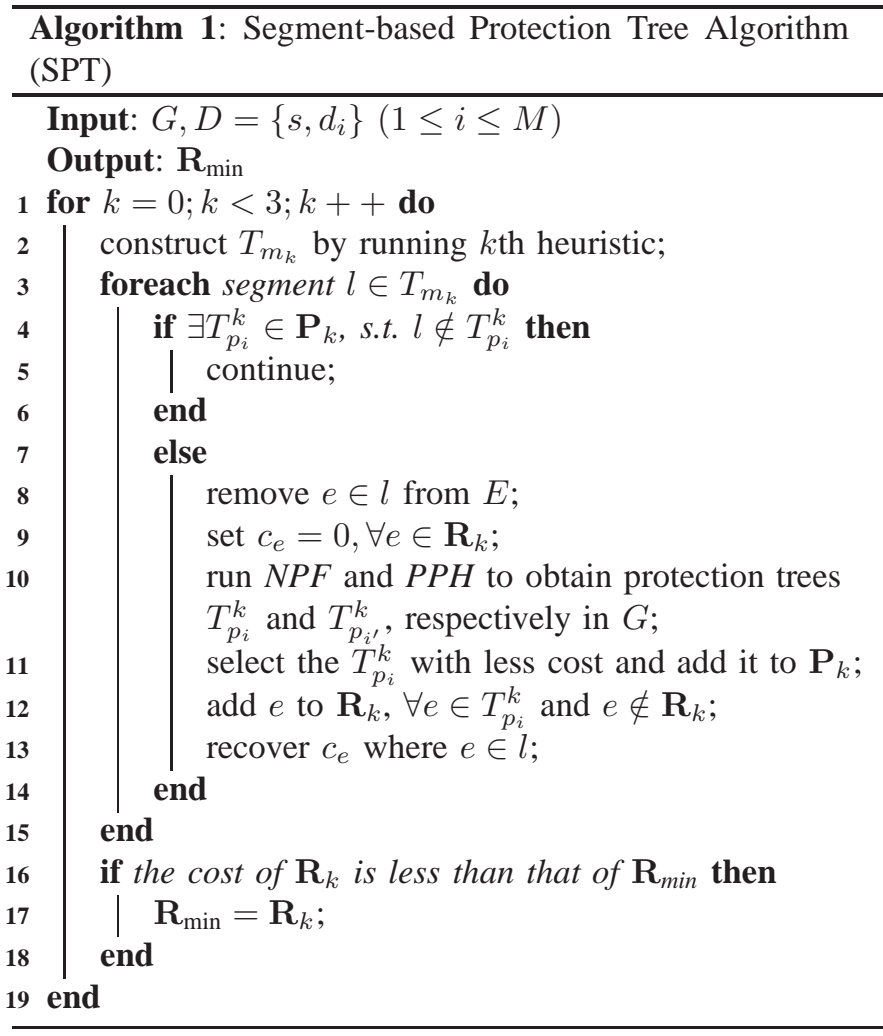

link $e \in l$. If no such protection tree exists, a new protection tree needs to be provisioned. However, the new tree can share any link with all the established trees in $\mathbf{P}_{k}$ as well as the primary tree $T_{m_{k}}$ in the modified graph $G^{\prime}$ with removal of $l$. Hence, we set the cost of all links available for sharing as 0 . Then, algorithm NPF and PPH are executed to obtain the new protection trees $T_{p_{i}}^{k}$ and $T_{p_{i^{\prime}}}^{k}$ and the one with the less link cost will be selected and added into the protection tree set $\mathbf{P}_{k}$ in which the links that do not exist in final set $\mathbf{R}_{k}$ will also be added. In the final step, three final sets with three different primary trees are compared and we choose the one with the minimum cost, $\mathbf{R}_{\min }$, as our final survivable topology.

Since the number of links of a tree is less than $|V|$, in the worst case, the number of segments on a primary tree cannot exceed $|V|$. Therefore, the time complexity of heuristic SPT is $O\left(3 M|V|^{3}\right)$.

\section{ReCONFIGURATION CALCULATION}

Besides the network cost, the recovery time, referred to as the time period from the occurance of the failure to the restoration of the traffic, is another important criteria to evaluate the performance of a protection approach. The recovery process consists of several stages: failure detection, signaling transmission and switch reconfiguration, in which switch reconfiguration process consumes the most part of recovery time, since each reconfiguration takes 10 - 20s ms [14] depending on the technology used. Therefore, it is essential to figure out the average reconfiguration time upon any link failure in a network. 
Based on the SPT approach proposed in Section III, a multicast tree is provisioned first and then each segment on the tree will be protected by a protection tree. Thus, given a failure in a network, if this link happens to be used by the multicast tree, a protection tree will be activated to protect it. Accordingly, some nodes on the protection tree may be required to reconfigure the switches to reroute the traffic. The rule to determine whether a node needs to reconfigure its switch is whether this node receives the incoming traffic from a different node or forwards it to a different output node in the protection tree compared to that in the primary multicast tree.

In order to obtain the average number of reconfigurations upon any link failure that disrupts a given multicast service, we assume that the primary tree $T_{m}$ consists of $L$ links and upon the failure of link $e \in T_{m}$, a protection tree $T_{p_{i}}$ is activated and $r_{i}$ nodes on $T_{p_{i}}$ will reconfigure the switch. Therefore, the average number of reconfigurations given any link failure is denoted by:

$$
R_{\mathrm{avg}}=\frac{\sum_{e \in T_{m}} r_{i}}{L} \quad, \text { where } T_{p_{i}} \text { protects } e
$$

Based on the previous analysis, we propose Algorithm 2 to compute the average reconfiguration time with the application of SPT approach. Several symbols used in the algorithm are explained in Table II:

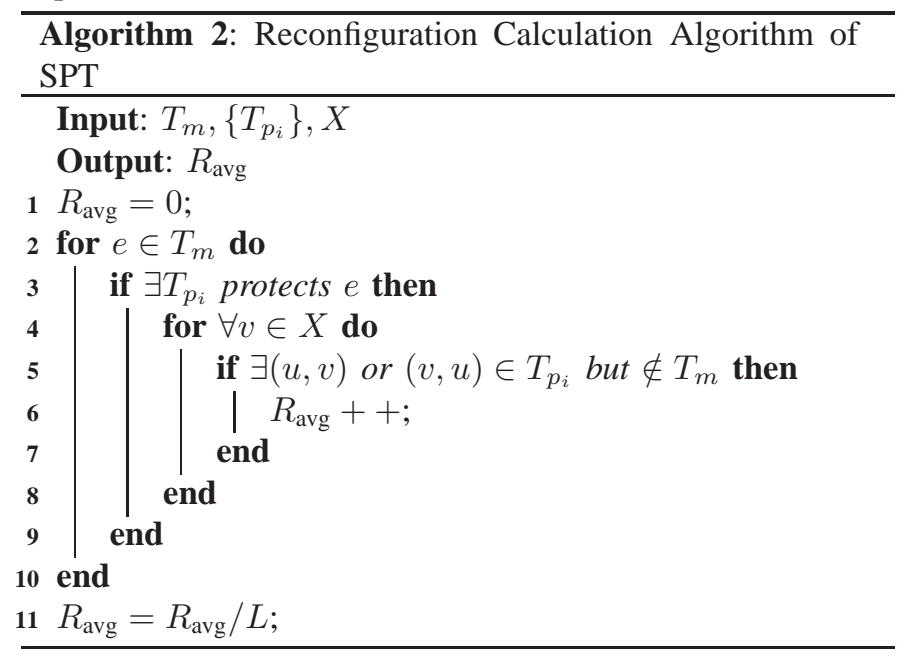

In the Algorithm 2, the set $X$ maintains all the potential nodes that may reconfigure the switch upon a link failure. Any node in the final survivable topology $\mathbf{R}$ has node degree at least 2 , since $\mathbf{R}$ is two connected. Except the source $s$, every node has at least a parent. If a node has node degree 2 , the incoming and outgoing links that the traffic passes through will always be fixed and there is no need for reconfiguration. Therefore, we only consider the nodes with node degree at least 3 along with the source and the destinations as the potential nodes. In the algorithm, line (4) checks whether node $v$ needs reconfiguration or not. If yes, line (5) increases the total number of reconfigurations. Therefore, the average number of reconfigurations is obtained by the total number divided by the total number of the links in the primary tree shown in line (11). The time complexity of Algorithm 2 is $O\left(L|V|^{2}\right)$.

TABLE II

LIST OF SYMBOLS

\begin{tabular}{ll}
\hline \hline Symbol & Meaning \\
\hline$L$ & $\begin{array}{l}\text { total number of links in the primary tree } T_{m} \\
\text { the set of nodes that consists of }\left\{s, d_{i}\right\} \text { and the nodes } \\
\text { that have node degree more than } 2 \text { in the final survivable }\end{array}$ \\
& $\begin{array}{l}\text { topology } \mathbf{R} \\
\text { the average number of reconfigurations given any single } \\
R_{\text {avg }}\end{array}$ \\
\hline
\end{tabular}

\section{Numerical Results}

In order to investigate the overall performances of the proposed multicast protection scheme, SPT, in our study, we consider two network topologies: USnet[2] and NSF network[15]. Each link is assigned a certain cost determined by the distance between two end nodes. USnet has a greater number of nodes, links and average node degree than NSF network.

The results consist of two parts. In the first part, we calculate the average cost of provisioning a given multicast session by using SPT in both network topologies. We will compare them with the best existing heuristics, OPP_SDP, as well as the optimal solution. In the second part, we compare the average number of reconfigurations between SPT and OPP_SDP upon any single-link failure.

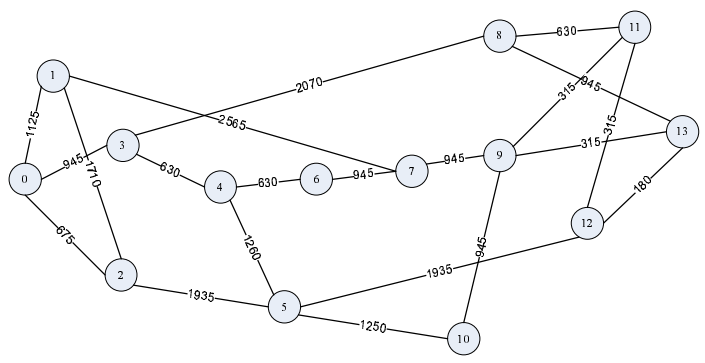

Fig. 2. NSF network (14 Nodes, 21 Links)

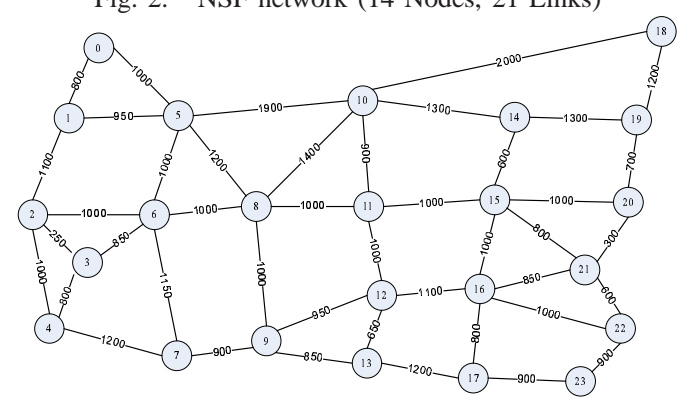

Fig. 3. USnet (23 Nodes, 43 Links)

\section{A. Total Network Cost}

We investigate the total link cost to route one multicast session and its protection trees in this part. A number of assumptions should be claimed as follows: 
TABLE III

THE COMPARISON OF AVERAGE NETWORK COST OF PROVISIONING A SURVIVABLE MULTICAST SESSION IN NSF NETWORK

\begin{tabular}{|c|c|c|c|c|c|c|c|c|c|c|c|}
\hline Session Size & 2 & 3 & 4 & 5 & 6 & 7 & 8 & 9 & 10 & 11 & 13 \\
\hline Optimal & 8835.5 & 10804.1 & 12537.2 & 13810.5 & 15097.2 & 16240.6 & 17152.1 & 18224.8 & 18984.2 & 19720.4 & 21164.9 \\
\hline & 8904 & 11021.8 & 13274.1 & 14563.4 & 15833.7 & 16899.3 & 17871.3 & 19415.3 & 19876.5 & 20938.9 & 22491.9 \\
\hline OPP_SDP & 8922.2 & 11292.3 & 13383 & 14757.7 & 16262.6 & 17420.4 & 18432 & 20039.9 & 20572.7 & 21648.6 & 23351.6 \\
\hline Saving Ratio (\%) & 0.20 & 2.45 & 0.82 & 1.33 & 2.71 & 3.08 & 3.14 & 3.22 & 3.50 & 3.39 & 3.82 \\
\hline
\end{tabular}

TABLE IV

THE COMPARISON OF AVERAGE NETWORK COST OF PROVISIONING A SURVIVABLE MULTICAST SESSION IN USNET NETWORK

\begin{tabular}{|c|ccccccccccc|}
\hline Session Size & 2 & 4 & 6 & 8 & 10 & 12 & 14 & 16 & 18 & 20 & 23 \\
\hline Optimal & 10839 & 15909.1 & 19696.7 & 22698.9 & 25518.4 & 28126.7 & 30491.4 & 32935.1 & 35209.61 & 37461.0 & 40838.6 \\
SPT & 11076 & 16233.5 & 19974 & 24002.5 & 27212.5 & 29638.5 & 32199 & 34914 & 36607.5 & 39493 & 42761 \\
OPP_SDP & 11393 & 16234 & 20319.5 & 24224.5 & 27649.5 & 30132.5 & 32830.5 & 35526.3 & 37366.5 & 39770.6 & 43307.5 \\
Saving Ratio (\%) & 2.86 & 0.01 & 1.73 & 0.92 & 1.61 & 1.67 & 1.96 & 1.75 & 2.07 & 0.70 & 1.28 \\
\hline
\end{tabular}

1) A network scenario is defined by one source and $M$ destinations and the source and destinations are randomly generated for each network scenario.

2) The traffic rate of each multicast session is one wavelength and the links of network topologies are uncapacitated.

3) For each network scenario, we run the simulation 200 times and take the average value.

Given fixed traffic pattern, we compare the average cost achieved by SPT scheme with OPP_SDP algorithm as well as the optimal solution solved by formulating the problem using Integer programming, which is also proposed in [2]. Tables III and IV illustrate the average cost of provisioning a multicast session obtained by different approaches in NSF and USnet networks, respectively, in which session size denotes the number of destination in a session and saving ratio reflects the cost saving ratio of heuristic SPT over OPP_SDP and is defined by $\left(C_{\mathrm{OPP}}\right.$ SDP $\left.-C_{\mathrm{SPT}}\right) / C_{\mathrm{SPT}}$.

It is clear that results produced by both SPT and OPP_SDP are close to the optimal solutions within 10\% in NSF network and $15 \%$ in USnet. However, SPT produces lower total cost than OPP_SDP approach in both network topologies. The saving ratio of SPT over OPP_SDP in NSF network is between $0.2 \%$ and $4 \%$, and the most saving ratios in USnet fluctuate between $1 \%$ and $2 \%$ with various session sizes. In NSF network, the advantage of SPT over OPP_SDP gradually increases as the session size increases, which is not the case in USnet. One of the reasons is that NSF network has averagely smaller node degree such that finding two link disjoint paths for each pair of source and destination conducted in OPP_SDP scheme may end up with long paths. However, SPT is not affected as much since different segments may share the same protection tree. The larger the session size is, the higher possibility that segments will share protection with one another. However, this feature cannot be applied to OPP_SDP scheme.

In USnet, the average node degree is higher and the distances between different pairs of nodes do not vary as much as in NSF network. The shortest path pair established earlier in OPP_SDP scheme may be shared by other source and destination pairs with higher probability. Therefore, the advantages of SPT scheme is not as significant as that in NSF network.

\section{B. Average Number of Reconfigurations}

We also studied the failure recovery performance in terms of average number of switch reconfigurations given any link failure in both NSF and USnet network topologies. The method of calculating the number of reconfigurations in the SPT scheme has been presented in Section IV. In OPP_SDP scheme, the shortest pair of paths between the source and each destination is constructed. We consider one as the primary path and another as the protection path. The combination of all the primary paths construct a primary multicast tree. We assume that when a link on the multicast tree fails, all the disrupted primary paths will be rerouted from the source to the corresponding destinations through the protection paths. Accordingly, the same reconfigurations rule described in STP can be applied here. Hence, we obtained the average number of reconfigurations of both protection schemes in NSF and USnet networks as shown in Fig.4 and 5. Each value is obtained by taking the average over 200 independent cases for each network scenario.

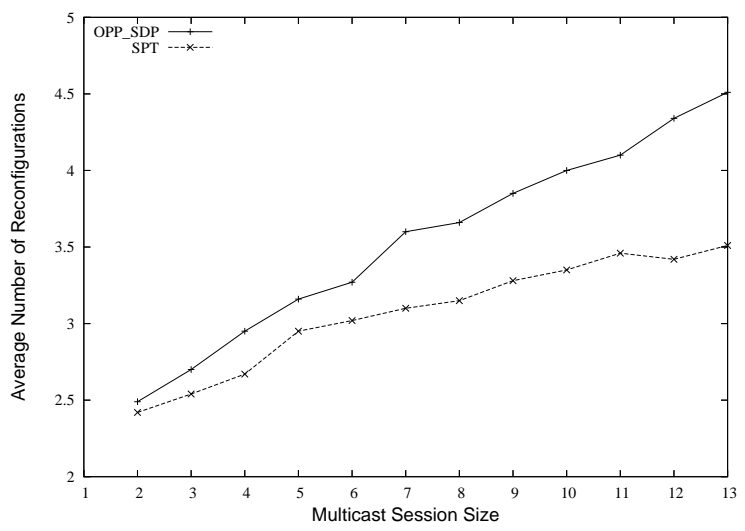

Fig. 4. Comparison of Average Number of Reconfigurations in NSF network 


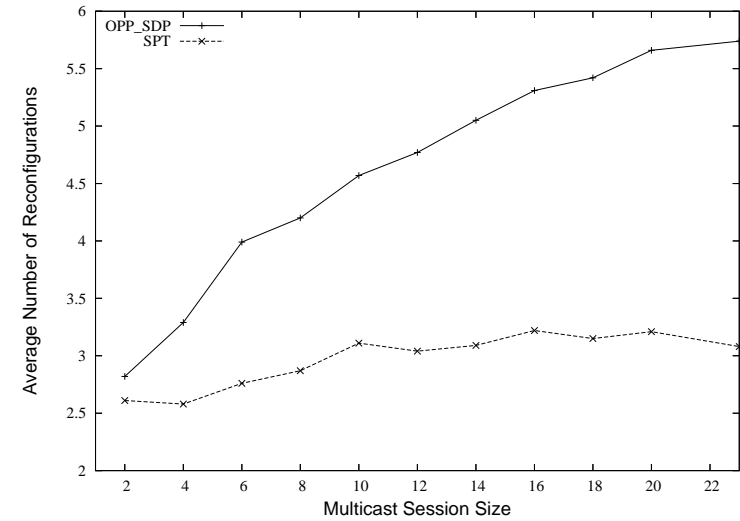

Fig. 5. Comparison of Average Number of Reconfigurations in USnet network

It is obvious that the average number of reconfigurations increases as the session size increases in both topologies due to the fact that final topology gets larger and denser and link sharing becomes more prevalent between different source and destination pair. Therefore, the average node degree of the survivable multicast session gets higher and more nodes will become potential switch nodes. Thus, more nodes will actually reconfigure their switch upon a link failure. However, the increase speed of OPP_SDP approach is much faster than SPT as the increase of the session size, because the larger number of destinations in the session results in more path pairs in the multicast topology and one link capacity may be shared by a large number of primary paths. Therefore, one link failure will disrupt more primary paths and cause more reconfigurations. As we can see in the figures that the performances of SPT and OPP_SDP are close when there are only two destinations. However, when they provision the broadcast services, the advantages of SPT over OPP_SDP reaches almost 30\% in NSF network and $86 \%$ in USnet.

However, SPT performs very well in USnet since the average number of reconfigurations grows very slowly as the session size increases. Since each protection tree is independent from one another and also from the multicast tree, each protection tree can share a large number of links with primary tree except the segment it protects, which means any link failure will not result in a significant change between the multicast tree and the corresponding protection, especially when the session size is very large. Therefore, only a limited number of nodes may need reconfiguration differing significantly from OPP_SDP scheme in the same scenario. In summary, SPT outperforms OPP_SDP in terms of the configuration time in all the network scenarios in our study and the advantages vary from $10 \%$ to $86 \%$.

\section{Conclusions}

We studied the problem of provisioning a survivable multicast session with protection against single link failures in a network with minimum resources, and proposed a heuristic algorithms, Segment-based Protection Tree (SPT). In SPT scheme, three primary multicast trees are established first by three different multicast provisioning approaches, NPF, PPH and DST, respectively, and then each segment of each primary tree is protected by a multicast tree, called protection tree, which is selected out of two candidates produced by NPF and $\mathrm{PPH}$, respectively. Each primary tree and its corresponding protection trees compose a survivable topology. We choose the one with minimum network cost as the final topology.

We studied the performance of SPT in terms of network cost and average number of reconfigurations. SPT uses only $10 \%$ extra cost over the optimal solution under all network scenarios considered and only $5 \%$ extra cost over the optimum when the session size is very small or large, such as unicast or broadcast, respectively. SPT scheme also outperforms OPP_SDP approach, the current most cost efficient algorithm, in terms of overall network cost by $0.2-4 \%$ in different traffic scenarios. We also investigated the recovery performance of SPT and compared the average number of reconfigurations with OPP_SDP scheme. The results show that SPT has a significant advantage over OPP_SDP and the average number of reconfigurations upon any link failure is $10-86 \%$ less than that of OPP_SDP in various network scenarios.

\section{REFERENCES}

[1] J. He, Gary Chan and Danny H.K. Tsang, "Multicasting in WDM networks," IEEE Commun. Surveys and tutorials, Dec. 2002.

[2] N. K. Singhal, L. H. Sahasrabuddhe, and B. Mukherjee, "Provisioning of survivable multicast sessions against single link failures in optical WDM mesh networks", J. Lightwave Technol, vol. 21, pp. 2587-2594, 2003.

[3] L. H. Sahasrabuddhe and B. Mukherjee, "Light trees: Optical multicasting for improved performance in wavelength routed networks," IEEE Commun. Mag., vol. 37, no. 2, pp. 67-73, Feb. 1999.

[4] M. Medard, S. G. Finn, R. A. Barry, and R. G. Gallager, "Redundant trees for preplanned recovery in arbitrary vertex-redundant or edge redundant graphs," IEEE/ACMTrans. Netw., vol. 7, no. 5, pp. 641-652, Oct. 1999.

[5] N. Singhal and B. Mukherjee, "Protecting multicast sessions in WDM optical mesh network," IEEE/OSA J. Lightwave Technol., vol. 21, no. 4, pp. 884-892, April 2003.

[6] H. Luo, L. Li, H. Yu and S. Wang, "Achieving shared protection for dynamic multicast sessions in survivable mesh WDM networks", IEEE J. on Sel. Area in Commun., Vol. 25, No. 9, Dec. 2007.

[7] N. K. Singhal, C. Ou, and B. Mukherjee, "Cross-sharing vs. self-sharing trees for protecting multicast sessions in mesh networks,"Computer Networks., vol. 50, no. 2, pp. 200-206, 2006.

[8] F. Zhang, W. Zhong, and Y. Jin, "Optimizations of p-Cycle-based protection of optical multicast sessions," J. Lightwave Technol, vol. 26, No. 19, Oct. 2008.

[9] R. Koetter, M. Medard, "An Algebraic Approach to Network Coding", IEEE/ACM Trans. on Netw., Vol. 11, No. 5, Oct. 2003.

[10] S. L. Hakimi, "Steiners problem in graphs and its implications," Networks, vol. 1, no. 2, pp. 113-133, 1971.

[11] H. Takahashi and A. Matsuyama, "An Approximate Solution for the Steiner Problem in Graphs", Mathematica Japonica 6 (1980), 573-577.

[12] L. Kou, G. Markowsky and L. Berman, "A fast algorithm for Steiner trees". Acta Informatica 15 (1981), pp. 141-145.

[13] T. H. Corman, C. E. Leiserson, and R. L. Rivest, "Introduction to Algorithms," 2nd ed. Cambridge, MA: MIT Press, 2001.

[14] S. Thorpe, G. Edwards, and D. Stevenson, "Using Just-in-Time to Enable Optical Networking for Grids", Proc. IEEE Broadnets Workshop, Oct. 2004.

[15] S. Baroni, "Routing and wavelength allocation in WDM optical networks", PhD Thesis, University College London, May 1998, pp. 118. 